\title{
Analyse de la gélivité des bourgeons de vigne. Expérimentation in situ sur le vignole champenois
}

\author{
B Itier 1, D Flura 1, O Brun 2, J Luisetti 3, JL Gaignard 3, C Choisy 4, G Lemoine 4 \\ avec la collaboration technique de B Durand ${ }^{1}, \mathrm{~S}$ Masson ${ }^{1}, \mathrm{M}$ Devaux ${ }^{3}, \mathrm{JC}$ Lhotel 5 \\ 1 INRA, station de bioclimatologie, 78850 Thiverval-Grignon; \\ 2 GCEV MUMM vignobles et recherches, 11 avenue de Champagne, 51200 Epernay; \\ 3 INRA, station de pathologie végétale et phytobactériologie, route de Saint-Clément, Beaucouzé, 49000 Angers; \\ 4 Faculté de pharmacie, laboratoire de microbiologie, 52 rue Cognacq-Jay, 51096 Reims Cedex; \\ 5 INRA, station de bioclimatologie, domaine de Saint-Paul, BP 91, 84140 Montfavet, France
}

(Reçu le 12 juin 1990; accepté le 2 janvier 1991)

\begin{abstract}
Résumé - Une étude expérimentale de la gélivité des bourgeons de vigne in situ a été entreprise sur le vignoble champenois (Vitis vinifera L Chardonnay) de 1987 à 1989 en utilisant l'analyse thermique différentielle dans des chambres de refroidissement. L'analyse de la gélivité a consisté à étudier l'influence sur la température de rupture de surfusion de facteurs tels que le stade phénologique, le mouillage des organes végétaux et la teneur en bactéries dites glaçogènes. Au vu des résultats il apparaît que stade phénologique et mouillage sont des variables déterminantes, tandis que l'action des bactéries est beaucoup plus ténue et difficile à interpréter. Les informations recueillies doivent permettre à terme d'élaborer un modèle probabiliste du risque de rupture de surfusion dans le bourgeon.
\end{abstract}

\section{gel / sensibilité / bourgeon / Vitis vinifera = vigne / bactérie / phénologie / mouillage}

Summary - An analysis of sensitivity to spring frost in vine buds. An experiment on sensitivity to spring frost in vine buds was undertaken in the champagne vineyard (Nitis vinifera L Chardonnay) during the springs of 1987-1989 by means of differential thermal analysis (first exotherm) performed in freezing chambers, providing either convective cooling (on vine directly in the field) or radiative cooling on twigs. The sensitivity of buds was analysed in terms of bud phase, surface wetness and ice nuclei bacteria (Pseudomonas syringae) concentration. Figure 1 shows the evolution with phenological phase of mean freezing point of dry buds for 3 yr independently of ice nuclei concentration. Figure 2 reports this freezing point for dry vine buds which contain $<10^{3}$ bacteria, ie quasi-free of ice nuclei. Figure 3 reports for stage 9 of Eichhhorn and Lorenz the freezing point of dry buds versus ice nuclei concentration. The evolution of the mean freezing point at different stages is plotted on figure 4 versus mean ice nuclei bacterial concentration for both dry and wetted vine buds. From this figure, one can see that frost sensitivity of buds is increased not only by phenology but also by wetness of bud surface. On the contrary, the influence of ice nuclei bacteria is not so obvious. It seems that this bacterial influence could be different depending on the origin of the bacteria. In figure 5 at stage 7 , the freezing point of contaminated buds $(x)$ is higher than that of buds which contain the same amount of bacteria $(\Delta)$. The only minor effect of natural ice nuclei bacteria concentration on buds was observed on the percentage of surviving buds for a given temperature : the concentration of bacteria observed on killed buds $(\square)$ is a little higher in figure 6 than those in surviving buds (+).

\section{frost $/$ sensitivity $/$ bud $/$ Vitis vinifera $=$ vine $/$ bacteria $/$ phenology $/$ wetness}

\section{INTRODUCTION}

Une étude intégrée des gelées de printemps doit mettre en regard de prévisions météorologiques, des seuils de sensibilité des organes végétaux, afin de pouvoir déterminer s'il est utile ou non de mettre en œurre des moyens de lutte contre le refroidissement nocturne. La prévision de l'évolution de la température au cours d'une nuit de refroidissement a fait au cours des dernières an- nées un progrès certain (Cellier, 1982; Cellier et Itier, 1984; Cellier, 1984; McVeigh, 1988). Il était important de faire progresser la connaissance dans le domaine du déterminisme de la gélivité, depuis la mise en évidence de l'aptitude de certaines bactéries associées aux plantes à entraîner la rupture de surfusion de solutions à des températures négatives proches de $-2{ }^{\circ} \mathrm{C}$ (Lindow, 1983). L'étude présentée ici a consisté à étudier, au cours de 3 saisons, l'évolution de la 
gélivité des bourgeons de vigne in situ en essayant de dégager des influences séparées des différents facteurs susceptibles d'intervenir dans ce déterminisme : la phénologie, l'état hydrique superficiel et la teneur en bactéries glaçogènes.

\section{MATÉRIEL ET MÉTHODES}

L'étude entreprise a été conduite sur le vignoble champenois du champagne Mumm, à Avize, dans la Marne; le cépage était du Chardonnay. Elle a été effectuée au cours des printemps 1987, 1988 et 1989. Les nuits de refroidissement radiatif naturel étant un phénomène rare malgré l'empreinte qu'elles laissent dans la mémoire paysanne, nous avons procédé à la réalisation de chambres de refroidissement permettant d'observer la sensibilité des bourgeons ayant parcouru leur cycle phénologique dans le vignoble jusqu'aux nuits d'expérimentations. Les chambres en question sont décrites en détail dans un article technique (Flura et al, 1991). Nous préciserons ici qu'une première catégorie de chambres dites convectives permettait de réaliser des refroidissements sur pied de vigne in situ au moyen de groupes froid montés sur chambres adaptables sur pied entier. Le refroidissement au moyen de congélateurs conduisant à ne jamais se trouver en présence d'air saturé ou sursaturé (dépôt de givre sur les plaques froides des congélateurs), une deuxième chambre, dite radiative, a été réalisée en transposant sur la chambre décrite par Marcellos (1981), un système de circulation à azote liquide, permettant de faire effectuer un refroidissement radiatif à partir d'un plafond porté à très basse température. Les chambres convectives ont permis d'effectuer un suivi de sensibilité tout au long des saisons de végétation 1987 et 1988 par répétition des expériences depuis le stade 3 jusqu'au stade 12 de la classification de Eichhorn et Lorenz (1977), tandis que l'année 1989 a été consacrée à des répétitions d'expériences beaucoup plus nombreuses focalisées sur les stades 3 et 9 respectivement. En 1987 et 1988, seules 3 chambres convectives étaient mises en œuvre; en 1989, aux 3 chambres précédentes, s'ajoutait la chambre radiative qui permet d'étudier la gélivité de bourgeons ayant subi un dépôt de rosée.
Au cours des 3 années, des mesures ont été effectuées sur 1405 bourgeons. Ces bourgeons ont été répartis en 4 classes : normaux, mouillés, contaminés et décontaminés. Le mouillage était réalisé artificiellement avant l'expérience, soit par dépôt d'une gouttelette d'eau sur bourgeons précoces, soit par pulvérisation d'eau distillée sur les feuilles à partir du stade 9 . La contamination a été réalisée par piqûre ou aspersion, $48 \mathrm{~h}$ avant l'expérience, au moyen de suspension bactérienne à $10^{8}$ bactéries $/ \mathrm{ml}$. La décontamination résultait d'un badigeonnage ou d'une pulvérisation $24 \mathrm{~h}$ avant l'expérience au moyen de kasumine à $2 \%$ de kasugamicine (Hakko chemical Industry Co, Ltd, Tokyo, Japon). Les chiffres correspondant à chaque lot sont portés tableau I. Les mesures ont été effectuées sur des bourgeons sur pied ou sur sarment afin de déterminer la différence systématique éventuelle de gélivité dès lors que les sarments étaient détachés du pied. En effet, dans la chambre radiative, il n'était possible d'effectuer des mesures que sur des sarments coupés. De manière à obtenir des résultats transposables aux conditions extérieures, les vitesses de refroidissement étaient programmées de telle sorte qu'elles soient identiques à celles que l'on observe à l'extérieur $\left(1-3^{\circ} \mathrm{C} / \mathrm{h}\right.$ au maximum). Au cours d'un refroidissement, l'ensemble des bourgeons observés étaient piqués au moyen de fins thermocouples de cuivre-constantan (diamètre $\approx 0,1 \mathrm{~mm}$ ) montés en différentiel par série de 10 , reliés à un organe végétatif $\mathrm{sec}$ tel qu'un morceau de vieux sarment (précision de mesure $\approx 0,2^{\circ} \mathrm{C}$ ). Pour valider cette technique, on avait préalablement vérifié, par des observations à la loupe binoculaire 5-7 h après l'arrêt du refroidissement, que les pourcentages de bourgeons morts étaient identiques à différentes températures seuils comprises entre $-4^{\circ}$ et $-8^{\circ} \mathrm{C}$, que l'on ait affaire à des bourgeons piqués avec du thermocouple ou à des bourgeons indemnes de piqûres. La vitesse de refroidissement était caractérisée par l'évolution de la température de ce morceau de sarment mort, et l'on enregistrait en permanence l'écart de température entre les différents bourgeons et ce morceau de sarment. Cet écart pouvant être négatif ou positif selon la position du bourgeon à l'intérieur de la chambre de refroidissement, mais ne dépassant généralement pas $1^{\circ} \mathrm{C}$, la mort par le gel était repérée au moyen de l'exotherme (brusque augmentation de température) consécutif à la réalisation de la chaleur latente de

Tableau I. Échantillonnage des bourgeons étudiés. a Chambres à refroidissement convectif; b chambre à refroidissement radiatif.

\section{Bourgeons}

Année Normaux Mouillés Contaminés Décontaminés

$\begin{array}{rrrrr}1987^{a} & 81 & 14 & 6 & 0 \\ 1988^{a} & 89 & 36 & 16 & 17 \\ 1989^{a} & 525 & 132 & 72 & 34 \\ 1989^{b} & 332 & 0 & 34 & 17\end{array}$


congélation lors de la rupture de surfusion (Annexe 1). Une évaluation de la correspondance entre présence d'un exotherme et mort du bourgeon a été effectuée en 1987 à partir d'observations à la loupe binoculaire 5-6 h après que l'expérience eut été terminée. En plus des enregistrements de température, un certain nombre de notations étaient effectuées : elles concernaient le stade phénologique (classification de Baggiolini, 1952, ou Eichhorn et Lorenz, 1977), la taille du bourgeon, son poids et les communautés de pieds et de sarments des différents bourgeons. De même, à côté de la lecture du point de rupture de surfusion obtenu grâce à l'exotherme, la lecture du point de congélation était réalisée lorsque les bourgeons étaient suffisamment gros pour qu'un plateau correspondant à cette valeur soit lisible. Par ailleurs, tous les bourgeons ayant servi à l'expérimentation étaient prélevés de manière à effectuer une estimation de leur teneur en bactéries Pseudomonas syringae (par $\mathrm{g}$ et par bourgeon), ces dernières étant susceptibles d'avoir un pouvoir glaçogène augmentant la sensibilité au gel, tout autre facteur étant constant (Lindow, 1983; Luisetti et Gaignard, 1985). Parallèlement aux mesures de teneur en bactéries, une température de prise en glace du broyat de bourgeons était réalisée au moyen d'un cryostat, à partir de descentes en température de $1^{\circ} \mathrm{C}$ toutes les $5 \mathrm{~min}$.

\section{RÉSULTATS}

L'étude effectuée en 1987 a permis de constater que $96,4 \%$, des bourgeons présentant un exotherme étaient morts. Cette bonne correspondance a permis d'utiliser au cours des années suivantes l'égalité entre présence d'exotherme et mort par le gel.

La figure 1 permet de voir que la moyenne des points de rupture de surfusion (Annexe 1) est très proche d'une année à l'autre pour une variété donnée à même stade phénologique (pour les lecteurs peu familiarisés avec la notation des stades employée, on a les équivalences suivantes : stade 3 Eichhorn-Lorenz $=$ stade $B$ Baggiolini $=$ "bourgeon dans le coton"; stade 9 EichhornLorenz = stade $E$ Baggiolini $=$ «2-3 feuilles étalées"). Elle permet par ailleurs de valider a posteriori les résultats de 1987 et 1988 obtenus au moyen d'une chambre à refroidissement convectif à partir de la constatation faite en 1989 des identités de résultats obtenus en chambres à refroidissement radiatif et convectif. De plus, l'année 1989 a fourni l'occasion d'une comparaison de pourcentage de bourgeons gelés au cours d'une nuit de refroidissement radiatif naturel avec une expérience conduite la même nuit au sein de chambres de refroidissement. L'égalité des pourcentages ( $11 \%$ à l'extérieur et $15 \pm$ $4 \%$ à l'intérieur) permet de penser que la sensibilité à la survie dans les chambres traduit bien celle qui aurait été obtenue si le nombre de nuits de refroidissement radiatif avait été suffisant pour pouvoir envisager des expériences sans refroidissement artificiel.

Des expériences conduites par ailleurs au CNRS (Leddet et Dereuddre, 1991) sur bourgeons prélevés, ont permis de constater que si les vitesses de refroidissement plus rapides (5$20^{\circ} \mathrm{C} / \mathrm{h}$ ) conduisaient à des seuils de gélivité plus bas de $2-3^{\circ} \mathrm{C}$ environ, l'évolution en fonction du stade phénologique était tout à fait semblable. Les résultats portés sur la figure 1 contiennent des informations relatives non seulement aux stades phénologiques, mais également à des variables susceptibles d'avoir provoqué les évolutions de sensibilité (teneur en bactéries, mouillage, etc). Afin de faire la part de chacun des facteurs d'influence, une étude avec variables séparées a été effectuée en 1988 et 1989 en séparant les bourgeons mouillés des non mouillés, et en étudiant l'évolution du point de rupture de surfusion en fonction du stade phénologique par classe de bactéries. On peut voir sur la figure 2 l'augmentation de la température de rupture de surfusion avec le stade phénologique pour des teneurs en bactéries inférieures à $10^{3}$ bactéries par bourgeon ${ }^{*}$, à tous les stades. L'influence des bactéries seules a pu être étudiée à partir de l'évolution du point de rupture de surfusion pour un stade donné. Cette

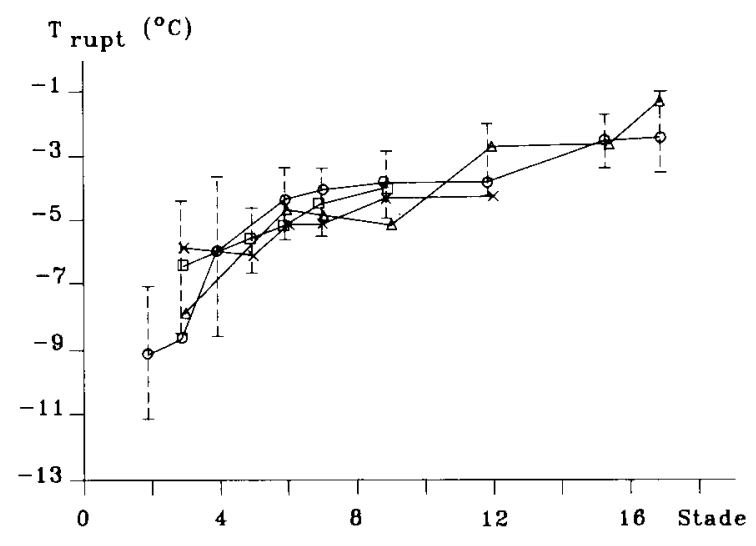

Fig 1. Évolution en $1987(\Delta-\Delta), 1988(O-O)$ et 1989: ( $\square-\square)$ chambres radiatives et $x-x$ chambres convectives), de la moyenne des températures de rupture de surfusion des bourgeons de Chardonnay (Vitis vinifera) en fonction du stade phénologique (classification de Eichhorn-Lorenz). La barre verticale en tiretés indique à chaque stade l'intervalle: moyenne \pm écart type pour l'ensemble des 3 années.

\footnotetext{
* Ce seuil est proche de la limite d'observation comme on peut le constater en figure 3.
} 


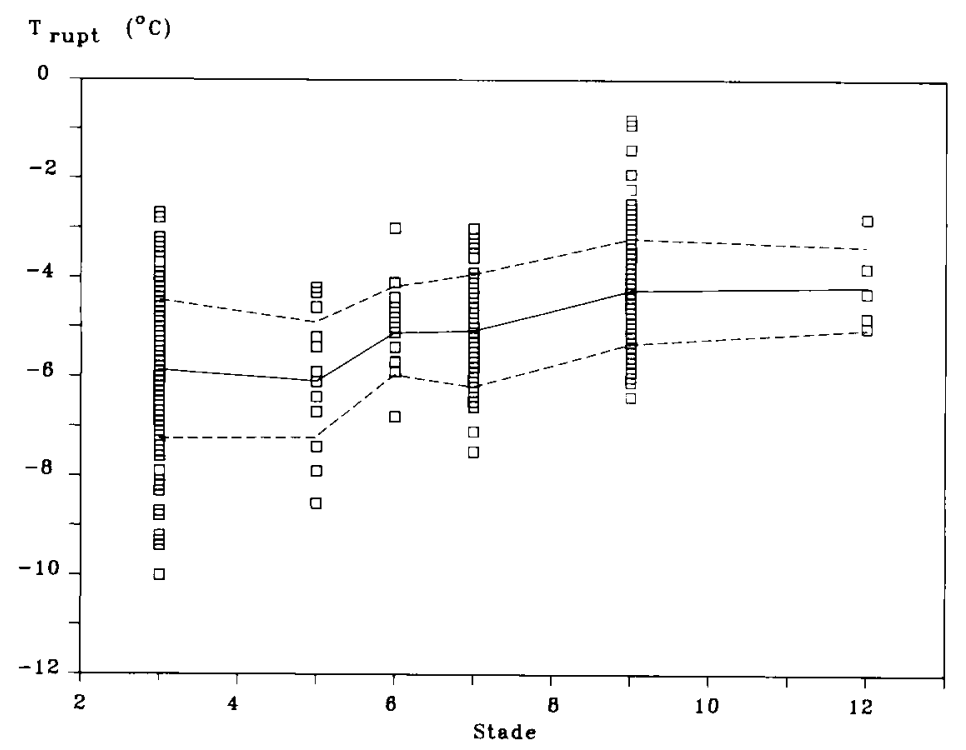

Fig 2. Évolution en 1989 des températures de rupture de surfusion de bourgeons de Chardonnay en fonction du stade phénologique pour des populations de bactéries (Pseudomonas syringae) inférieures à $10^{3}$ bactéries par bourgeon; - - : moyenne; - - - : moyenne \pm écart type.

étude a été effectuée en partant, soit de la teneur en bactéries/g soit de la teneur en bactéries par bourgeon, ce dernier représentant l'espace fermé au sein duquel un événement de prise en glace, susceptible d'être provoqué par un noyau glaçogène, pouvait avoir eu lieu. Au vu des résultats, il semble que seule la teneur par bourgeon puisse être susceptible de provoquer une légère différence dans les seuils de sensibilité.

La figure 3 montre l'évolution des températures de prise en glace pour des bourgeons sur pied au stade 9 en 1988 et 1989 en fonction de la teneur en Pseudomonas syringae. On constate que tant la moyenne que la dispersion semblent constantes dans toute la gamme des te${ }^{\mathrm{T}}$ rupt $\left({ }^{\circ} \mathrm{C}\right)$

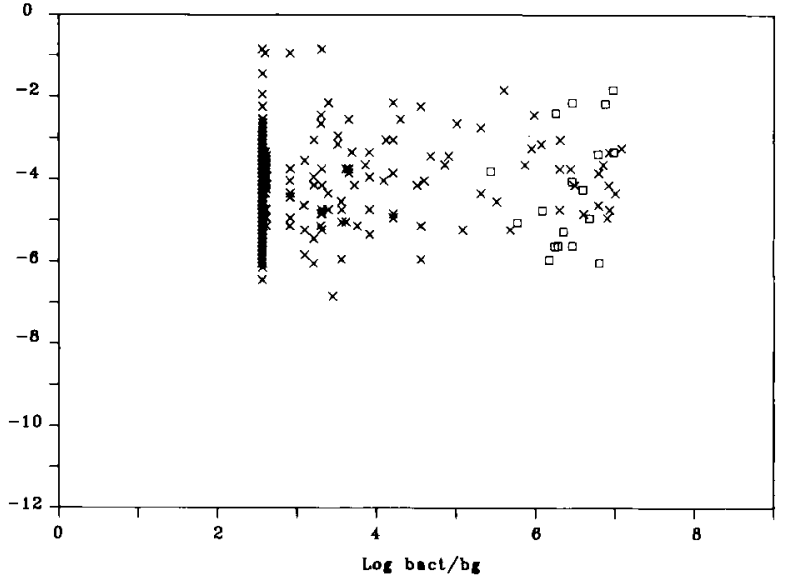

Fig 3. Évolution en 1988 (D) et 1989 (x) de la température de surfusion de bourgeons de Chardonnay en fonction de la teneur en bactéries/bourgeon pour le stade 9 de la classification de Eichhorn-Lorenz. neurs en bactéries. Les résultats obtenus aux autres stades phénologiques n'indiquent pas davantage qu'au stade 9 une influence marquée de la teneur en bactéries sur la température de rupture de surfusion.

La figure 4 illustre l'influence du mouillage sur la sensibilité au gel. Quel que soit le stade phénologique, le mouillage des bourgeons a pour effet de remonter nettement la température de rupture de surfusion. Le mouillage rend la température de rupture de surfusion supérieure à $-4^{\circ} \mathrm{C}$ à tous les stades phénologiques, même pour de faibles valeurs de teneurs en bactéries

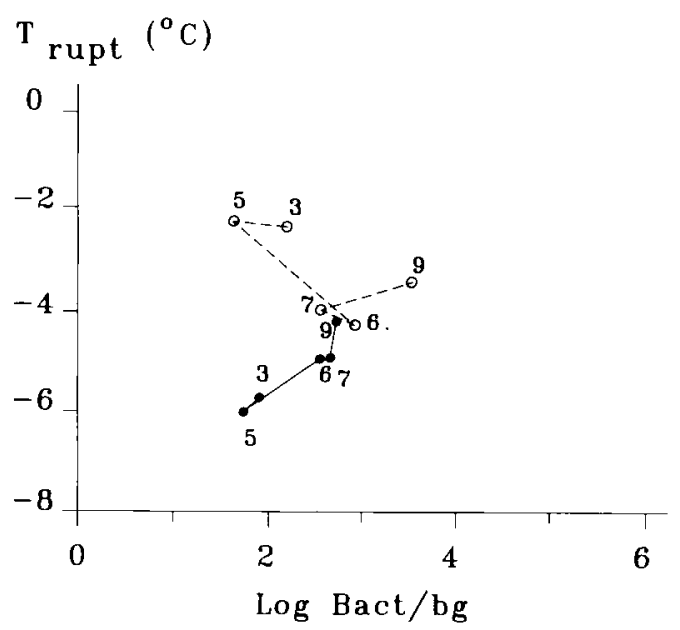

Fig 4. Évolution de la moyenne des températures de rupture de surfusion en fonction de la teneur en bactéries par bourgeon et du stade phénologique selon que les bourgeons de Chardonnay sont secs (-) ou mouillés (O--O). Les chiffres indiqués correspondent aux numéros des stades phénologiques. 
glaçogènes. Ceci justifie pleinement la règle retenue par les utilisateurs de moyens de lutte par aspersion et qui consiste, contrairement aux autres techniques de lutte, à ne pas prendre en compte la précocité du stade dans le seuil de déclenchement.

La figure 5 semble indiquer que la localisation des bactéries a une influence sur la température de rupture de surfusion. En effet, à un stade donné, pour une teneur en bactéries égale, le point de rupture de surfusion est déplacé de façon significative, selon que ces bactéries sont présentes naturellement, ou bien peuvent avoir une origine extérieure par contamination. Ceci semble confirmé par le lien entre teneur en bactéries et point de rupture de surfusion, lorsque l'on a nettoyé la surface de l'organe au moyen d'un désinfectant.

L'absence apparente d'action des bactéries, telle qu'elle apparaît sur la figure 3 , ne conduit pas à conclure à une indépendance entre teneur en bactéries et risque de gel. Ceci peut être illustré par la figure 6 où l'on a simulé une gelée à $-3,5^{\circ} \mathrm{C}$ et où l'on observe que la teneur en bactéries des bourgeons ayant gelé est légèrement supérieure à celle des bourgeons saufs. Sans être un facteur déterminant, ce type de résultat indique que la teneur en bactéries Pseudomonas syringae aggrave légèrement les risques. Par ailleurs, il semble qu'une teneur en Pseudomonas syringae plus élevée, sans modifier énormément les moyennes de températures de rupture de surfusion, remonte systématiquement les va-

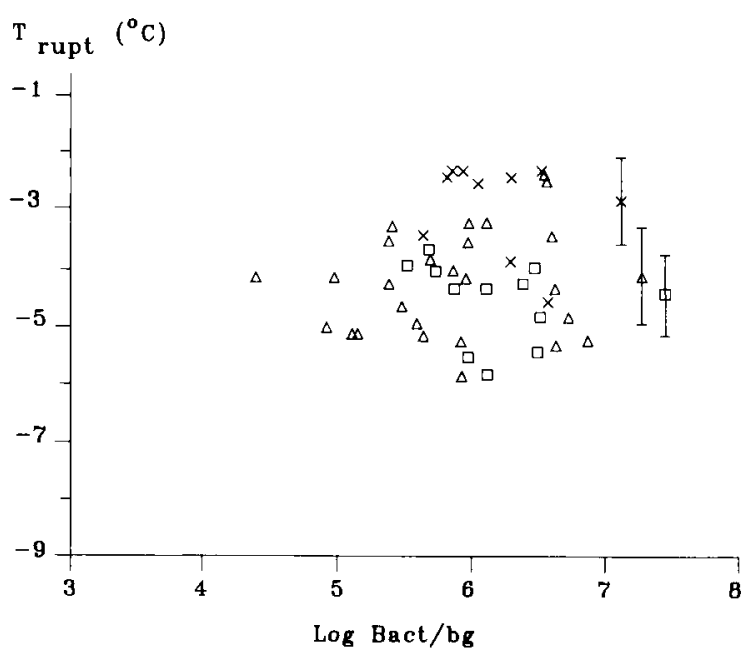

Fig 5. Température de rupture de surfusion de bourgeons de Chardonnay en fonction de la teneur en bactéries par bourgeon au stade 7 en 1988 selon que les bactéries sont constitutives $(\Delta)$, peuvent provenir d'une contamination $(x)$ ou qu'il $y$ a eu décontamination externe ( $\triangle$ ). On a porté, à droite de la figure, les moyennes \pm écart type de chaque catégorie.

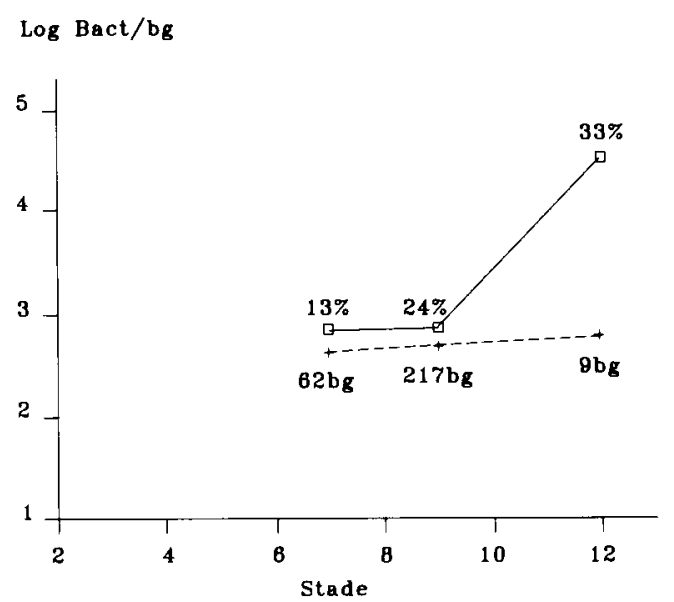

Fig 6. Comparaison des teneurs en bactéries de bourgeons gelés $(\square-\square)$ ou non gelés $(++)$ au seuil de $-3,5^{\circ} \mathrm{C}$. Les chiffres indiquent le pourcentage de bourgeons gelés et le nombre total analysé à un stade donné.

leurs minimales et ce, essentiellement au stade précoce. Ainsi, alors que la valeur minimale de températures de rupture de surfusion observée au stade 3 est proche de $-10^{\circ} \mathrm{C}$ en l'absence de bactéries (fig 2), elle remonte aux alentours de $-7^{\circ} \mathrm{C}$ pour des teneurs en bactéries supérieures à $3.10^{4}$. L'action des bactéries pourrait bien alors apparaître comme étant un facteur important sur le pourcentage de bourgeons survivant en cas de fortes gelées à un stade précoce.

\section{CONCLUSION}

L'étude conduite a tout d'abord montré l'utilité de l'approche par refroidissement artificiel dans la mesure où celle-ci permet de générer un nombre suffisant de cas pour pouvoir effectuer une analyse statistique des facteurs d'influence, contrairement aux situations de refroidissement réel qui, de par leur rareté, ne permettent pas d'obtenir des conclusions significatives. D'ores et déjà, l'ensemble des expériences permet de donner une valeur de seuil de gélivité à chaque stade et un écart type à cette valeur pour le vignoble champenois de la côte des Blancs, ce qui devrait donner une assise objective à des critères de déclenchement de moyens de lutte contre le gel. Pour ce qui concerne l'action des bactéries glaçogènes, il va sans dire que cette étude a permis d'établir que tout ne pourrait pas être résolu demain par le seul traitement antibactérien. Au vu de la faible action relative des bactéries en conditions naturelles, il est impor-

\footnotetext{
- Ce seuil est proche de la limite d'observation comme on peut le constater en figure 3.
} 
tant de poursuivre l'effort d'amélioration de la prévision et des moyens de lutte, car, en présence de bactéries ou non, il est établi que le seuil de gélivité remonte de valeurs inférieures à $-8^{\circ} \mathrm{C}$ au débourrement, à des valeurs voisines de $-3{ }^{\circ} \mathrm{C}$ au mois de mai. Une analyse fine de l'action des bactéries reste à entreprendre. II semble, d'ores et déjà, que ces actions soient significativement différentes selon que l'on a affaire à des organes ouverts comme les feuilles, ou de type fermé comme les bourgeons à des stades précoces. Ceci peut être illustré par la différence d'action des bactéries observée sur plant de tabac où il est évident que les feuilles de tabac prendront en glace si on a pulvérisé des suspensions de bactéries glaçogènes (Paulin et Luisetti, 1978), et celles observées in situ sur des bourgeons de vigne.

À côté du lien avec les études de déterminisme à l'échelle fine entreprises au CNRS (Leddet et Dereuddre, 1991), l'ensemble des résultats accumulés doit permettre d'effectuer une analyse de ce même déterminisme à l'échelle macroscopique à partir de modèles analogues à ceux qui ont été établis pour prévoir la prise en glace de gouttelettes d'eau à l'intérieur de nuages (Bigg, 1953). Cette analyse devrait conduire à expliquer la fourchette de température de rupture de surfusion observée à stade précoce pour de faibles teneurs en bactéries.

\section{REMERCIEMENTS}

Cette étude a été réalisée dans le cadre du programme de recherche financé par la Commission nationale des calamités agricoles que les auteurs remercient.

\section{ANNEXE 1, DÉFINITIONS}

Point de congélation : température à laquelle un corps passe de l'état solide à l'état liquide.

Température de rupture de surfusion (ou point de surfusion) : température inférieure au point de congélation, à laquelle un corps passe de la phase liquide à la phase solide. Si le volume est important, le point de surfusion est égal au point de congélation. Sur de faibles volumes (quelques $\mathrm{mm}^{3}$ ) d'eau pure, il peut être très inférieur (jusqu'à $-40{ }^{\circ} \mathrm{C}$ dans les nuages convectifs).
Aux températures intermédiaires, le liquide est dit surfondu. C'est un état métastable très fragile susceptible d'être interrompu par des agents nucléateurs de cristaux.

\section{RÉFÉRENCES}

Baggiolini M (1952) Les stades repères dans le développement annuel de la vigne et leur utilisation pratique. Rev Rom Agric VIII, 1

Bigg EK (1953) The supercooling of water. Proc Phys Soc B 66, 688-694

Cellier P (1982) Contribution à la prévision des températures minimales nocturnes en conditions de gelées de printemps. Étude de l'évolution des températures de l'air et du sol au cours de la nuit. Thèse de docteur ingénieur Sciences agronomiques, INA PG, $139 p$

Cellier $P$ (1984) Une méthode simple de prévision des températures de l'air et de surface du sol en conditions de gelées radiatives. Agronomie 4, 741-747

Cellier P, Itier B (1984) Un modèle de prévision de la température minimale nocturne sous abri et à la surface du sol en conditions de gelées radiatives. J Rech Atmos 1, 11-22

Eichhorn KW, Lorenz DH (1977) Phänologische Entwicklungsstadien des Rebe. Nachrichtenbl Dtsch Pflanzenschutzdienstes (Braunschw) 29, 119-120

Flura D, Itier B, Brun O, Durand B, Masson S (1991) Mise au point de chambres de refroidissement pour l'étude de la gélivité des bourgeons de vigne. Agronomie (à paraître)

Leddet C, Dereuddre J (1991) Mécanismes d'action du gel sur les organes végétaux. Communication au Séminaire "Le gel en agriculture", INRA, Paris 21-22 novembre 1989 (sous presse)

Lindow SE (1983) The role of bacterial ice nucleation in frost injury of plants. Annu Rev Phytopathol 21, 363-384

Luisetti J, Gaignard JL (1985) Les bactéries glaçogènes et les gelées de printemps. CR $5^{\circ}$ colloque recherches fruitières, INRACITFL, Bordeaux, Novembre 1985, 67-73

Mac Veigh (1988) Prévision des gelées radiatives. Rapport interne du service de météorologie interrégionale du Sud-Ouest, Bordeaux, $27 \mathrm{p}$

Marcellos $H$ (1981) A plant freezing chamber with radiative and convective energy exchange. $J$ Agric Eng Res 26, 403-408

Paulin JP, Luisetti J (1978) Ice nucleation activity among phytopathogenic bacteria. Proc 4th Int Conf Plant Path Bact, Angers 1978. Gibert-Clairey, Tours, France, vol II, 725-731 